Rafael de Assis da Silva1,2

Ohttps://orcid.org/0000-0002-5466-819

Marcelo Baggi Tancini²

Ohttps://orid.org/0000-0002-7417-4877

Elie Cheniaux ${ }^{3,4}$

Ohttps://orcid.org/0000-0003-4769-2684

Daniel C. Mograbi ${ }^{2,5}$

Ohttps://orcid.org/0000-0002-4271-2984

\section{Metacognição no transtorno bipolar: uma revisão sistemática}

\author{
Metacognition in bipolar disorder: a systematic review
}

DOl: $10.1590 / 0047-2085000000264$

\section{RESUMO}

Objetivo: Realizar uma revisão sistemática investigando a metacognição no transtorno bipolar (TB). Os objetivos secundários incluem explorar os correlatos clínicos e sociodemográficos da metacognição no TB e como a metacognição varia de acordo com o estado afetivo, estabelecer uma comparação com outros transtornos mentais e investigar se as intervenções metacognitivas no TB são eficazes ou não. Métodos: Realizou-se uma revisão sistemática da literatura científica sobre a metacognição em pacientes com TB. Foram buscados estudos clínicos originais sobre o tema nas bases de dados Medline, ISI, PsycINFO e SciELO. Os termos de busca empregados foram: "metacognition" OR "metacognitive" $O R$ "metamemory" AND "bipolar" OR "mania" OR "manic". Resultados: Foram selecionados nove artigos. A metacognição parece estar mais prejudicada no TB do que em controles e menos prejudicada do que na esquizofrenia. Por sua vez, parece não haver diferença entre bipolares e deprimidos unipolares quanto à capacidade metacognitiva. Maior nível educacional e maior duração da doença parecem estar associados a uma melhor capacidade metacognitiva, enquanto a maior gravidade dos sintomas de TB está associada a uma pior metacognição. O treinamento metacognitivo em pacientes com TB é uma perspectiva clínica promissora. Conclusão: Os estudos sobre metacognição no TB são escassos, mas a literatura existente indica possíveis fatores clínicos e sociodemográficos associados a pior metacognição no transtorno, sugerindo também que intervenções terapêuticas metacognitivas podem ser clinicamente relevantes para o manejo do TB.

\section{PALAVRAS-CHAVE}

Metacognição, metacognitivo, metamemória, bipolar, mania.

\section{ABSTRACT}

Objective: To perform a systematic review investigating metacognition in bipolar disorder (BD). Secondary objectives include exploring clinical and sociodemographic correlates of metacognition in $\mathrm{BD}$, how metacognition varies according to affective state, establishing a comparison with other mental disorders, and investigating whether metacognitive interventions in BD are effective or not. Methods: A systematic review of the scientific literature on metacognition in BD patients was carried out. Original clinical studies on the subject were searched in the Medline, ISI, PsycINFO and SciELO databases. The search terms included were: "metacognition" OR "metacognitive" OR "metamemory" AND "bipolar" OR "mania" OR "manic". Results: A total of nine articles were selected. Metacognition appears to be more impaired in BD than in controls, but less impaired than in schizophrenia. There seems to be no difference between bipolar and unipolar depression regarding metacognitive capacity. Higher educational level and longer duration of illness seem to be associated with better metacognitive capacity, while higher severity of BD symptoms is linked to worse metacognition. Metacognitive training in BD patients is a promising clinical perspective. Conclusion: Studies on metacognition in BD are scarce, but the existing literature indicates potential clinical and sociodemographic factors associated with poorer metacognition in the disorder, also suggesting that metacognitive therapeutic interventions may be clinically relevant for the management of BD.

\section{KEYWORDS}

Metacognition, metacognitive, metamemory, bipolar, mania. 


\section{INTRODUÇÃO}

A metacognição é um aspecto intimamente vinculado à consciência mais ampla de dificuldades e da presença de alterações mórbidas'. Metacognição pode ser definida como a capacidade de regular, controlar e monitorar as habilidades cognitivas, representando, em última instância, quão bem conhecemos nossas capacidades cognitivas². Essa capacidade amadurece gradualmente com o desenvolvimento normal $^{3}$, resultando em um modelo metacognitivo dinâmico e pessoal que inclui crenças sobre pensamentos ${ }^{4,5}$. As crenças metacognitivas podem levar à disfunção psíquica caso contribuam para interpretações equivocadas de pensamentos, objetivos inatingíveis ou recursos cognitivos enviesados. Exemplos de tais crenças são: "alguns pensamentos podem me fazer enlouquecer", "eu deveria estar no controle de meus pensamentos o tempo todo" ou "eu preciso constantemente examinar meus pensamentos".

Uma pior capacidade metacognitiva tem sido associada a desfechos mais desfavoráveis em diferentes quadros clíni$\cos ^{6}$, incluindo menores habilidades para lidar com o estig$\mathrm{ma}^{7}$, buscar apoio social ${ }^{8}$, ter um bom funcionamento em ambiente de trabalho ${ }^{9}$, vincular de forma coesa eventos da $v^{1} a^{10}$ e experimentar motivação intrínseca ${ }^{11,12}$. Níveis mais baixos de metacognição também foram associados a maiores dificuldades em reconhecer desafios psicossociais ${ }^{13,14} \mathrm{e}$ maior duração de quadro psicótico não tratado ${ }^{15}$.

Déficits metacognitivos têm sido observados na esquizofrenia ${ }^{16-20}$ e estão ligados a piores resultados clínicos nesse grupo. Um prejuízo na metacognição também foi relatado em transtornos da personalidade e abuso de substâncias ${ }^{21-23}$. Pacientes com transtorno obsessivo-compulsivo ${ }^{24,25}$, transtorno de pânico ${ }^{25,26}$ e transtorno de ansiedade generaliza$\mathrm{da}^{26,27}$ apresentaram mais crenças negativas sobre preocupação com incontrolabilidade ou perigo e crenças sobre a necessidade de controlar pensamentos do que controles saudáveis, sugerindo que uma pior capacidade metacognitiva esteja correlacionada com os transtornos de ansiedade. Pacientes com depressão maior têm dificuldades em tomar decisões sociais com base no conhecimento metacognitivo ${ }^{28}$ e, em geral, são menos conscientes de seus próprios estados emocionais ${ }^{29}$.

Em contraste, pouca pesquisa sobre metacognição foi conduzida no transtorno bipolar (TB), apesar do fato de que a falta de conhecimento da cognição possa estar implicada no transtorno por várias razões. Embora os déficits cognitivos sejam mais graves na esquizofrenia, eles podem ser comparáveis aos do TB ${ }^{30,31}$. Além disso, como na esquizofrenia, pacientes bipolares apresentam prejuízos proeminentes na percepção clínica de sua doença e de seus sintomas ${ }^{32-34}$. Embora o prejuízo de insight seja maior durante os episódios afetivos $^{35}$, também pode ser observado em pacientes eutími$\cos ^{36,37}$. Dado que o insight sobre a doença é um constructo que envolve um elemento de autoconsciência, sugere-se que déficits metacognitivos, que também envolvem essa característica, estejam presentes no TB.

Alguns estudos mostraram que pacientes bipolares apresentam prejuízo na capacidade de avaliar os estados mentais

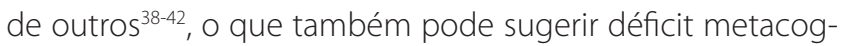
nitivo. Já um estudo longitudinal constatou que pacientes com TB recuperam sua capacidade de avaliação sobre os outros à medida que saem de algum episódio afetivo agu$\mathrm{do}^{43}$, o que pode indicar possível influência do estado afetivo atual sobre a metacognição. Um estudo conduzido por Lysaker et al. (2018) ${ }^{44}$ mostrou que pacientes com TB tinham habilidades metacognitivas semelhantes às de controles. Esses resultados se contrapõem aos achados de Popolo et al. $(2017)^{45}$ e Tas et al. (2014) ${ }^{46}$, que observaram maior prejuízo metacognitivo em pacientes bipolares do que em controles.

Os estudos sobre metacognição no TB parecem ser escassos e ainda com resultados contraditórios. Compreender a metacognição no TB pode apontar para a possibilidade de adaptar intervenções clínicas que se mostraram promissoras para melhorar a capacidade metacognitiva em pacientes com outras condições clínicas, como esquizofrenia ${ }^{47}$ e transtornos de personalidade ${ }^{48}$. Diante de tantas questões sobre a metacognição no TB e da relevância do tema, realizamos uma revisão sistemática investigando a metacognição no TB. Os objetivos secundários incluem explorar elementos sociodemográficos e clínicos associados à metacognição no TB, como a metacognição varia em função do estado afetivo e como o TB se compara com outros transtornos mentais em relação à metacognição e avaliar se a intervenção metacognitiva no TB é capaz de melhorar sua capacidade metacognitiva.

\section{MÉTODOS}

Realizou-se uma revisão sistemática da literatura científica sobre a metacognição em pacientes com TB. Essa revisão seguiu as orientações da Prisma Statement para revisões sistemáticas da literatura e metanálises de estudos que avaliam intervenções em saúde ${ }^{49}$. Foram buscados estudos clínicos originais sobre o tema, até dezembro de 2018, por um pesquisador psiquiatra. 0 processo de busca dos artigos ocorreu em dezembro de 2018. Foram utilizadas as bases de dados Medline, ISI, PsycINFO e SciELO. Não houve restrição quanto ao período da publicação. Os termos de busca empregados foram: "metacognition" OR "metacognitive" OR "metamemory" AND "bipolar" OR "mania" OR "manic".

Foram selecionados artigos originais e empíricos em que metacognição foi avaliada em uma amostra de pacientes com idade maior ou igual a 18 anos, de ambos os sexos, e que possuíssem diagnóstico de TB. Foram incluídos artigos com delineamento transversal e longitudinal publicados nas línguas inglesa, francesa, espanhola ou portuguesa. 
Os estudos deveriam utilizar uma escala, tarefa, treinamento ou algum item específico que aferisse a metacognição. Não houve qualquer limitação sobre os instrumentos que seriam aceitos para a avaliação da metacognição.

Foram excluídos nesta revisão artigos em que o TB não fosse o transtorno principal do paciente. Os estudos que apresentassem amostras contendo outros tipos de transtornos mentais além do TB foram excluídos quando apresentaram resultados que não eram específicos para o grupo de pacientes bipolares. Relatos de casos, cartas ao editor, estudos em crianças e adolescentes ou estudos com menos de 10 pacientes não foram selecionados. Artigos em duplicata tiveram uma de suas publicações excluídas da análise.

Um pesquisador realizou a extração dos dados e documentou o nome dos autores, o ano, o desenho do estudo, o tamanho da amostra, o instrumento de avaliação da metacognição, o objetivo e o resultado dos estudos. Não houve procura por estudos não publicados. Somente um juiz realizou o julgamento dos artigos que foram incluídos/excluídos.

Foi realizada uma análise descritiva dos estudos utilizando tabela e fluxograma, e uma discussão qualitativa dos achados foi realizada. Variáveis demográficas como idade, variáveis clínicas, como o estado afetivo atual, a comparação da metacognição entre TB e outros transtornos mentais, como esquizofrenia, e intervenção metacognitiva foram analisadas.

Para análise da qualidade dos artigos, foram utilizados critérios de Cochrane Handbook for Systematic Reviews of Interventions ${ }^{50}$ (ver tabela 1). O artigo que que preenchesse dois dos critérios estabelecidos com a resposta "não" ou três critérios com a resposta "não está claro" não seria enquadrado como um artigo de qualidade e, portanto, seria excluído. Dois pesquisadores foram responsáveis por essa análise.

Tabela 1. Critérios de avaliação de qualidade para inclusão de estudos

\begin{tabular}{|c|c|c|}
\hline & & $\begin{array}{c}\text { Julgamento } \\
\text { (sim/não/não } \\
\text { está claro) }\end{array}$ \\
\hline \multirow[t]{3}{*}{ Amostragem } & $\begin{array}{l}0 \text { design do estudo foi apropriado para } \\
\text { responder à questão da pesquisa? }\end{array}$ & \\
\hline & 0 método de amostragem foi apropriado? & \\
\hline & $\begin{array}{l}\text { Se aplicável, o grupo controle foi comparável } \\
\text { ao grupo experimental? }\end{array}$ & \\
\hline \multirow[t]{2}{*}{ Medição } & Foi usada uma medida adequada? & \\
\hline & $\begin{array}{l}\text { Potenciais variáveis intervenientes foram } \\
\text { medidas? }\end{array}$ & \\
\hline Análise & As análises foram apropriadas? & \\
\hline
\end{tabular}

\section{RESULTADOS}

Na busca inicial, encontrou-se um total de 21 referências no Medline, 48 no ISI, 39 na PsycINFO e 0 no SciELO, com diversas superposições entre essas bases de dados.
Após a leitura dos resumos das referências encontradas no Medline, selecionaram-se 12 artigos.

$\mathrm{Na}$ base ISI, após a leitura dos resumos dos artigos, selecionaram-se 15 artigos. Desses 15 artigos, 12 foram repetidos em relação à base Medline e, portanto, foram excluídos.

Na base PsycINFO, após a leitura dos resumos dos artigos, selecionaram-se nove artigos. Todos os nove já haviam sido citados na base Medline, e, portanto, foram excluídos.

Ao todo, foram selecionados 15 resumos das quatro bases pesquisadas. Esses 15 artigos foram lidos na íntegra. Seis artigos apresentaram amostras contendo outros tipos de transtornos mentais, além do TB, e não apresentaram resultados específicos para o grupo de pacientes bipolares, sendo, portanto, excluídos. Não foram encontrados artigos com menos de 19 pacientes ou que não tenham usado algum método de avaliação da metacognição. Ao final, foram selecionados nove artigos. O fluxograma de busca e seleção dos artigos aparece na figura 1.

Esses nove artigos foram analisados quanto a sua qualidade. Considerando os critérios adotados, nenhum dos artigos foi excluído.

Os estudos sobre metacognição no TB são apresentados na tabela 2.

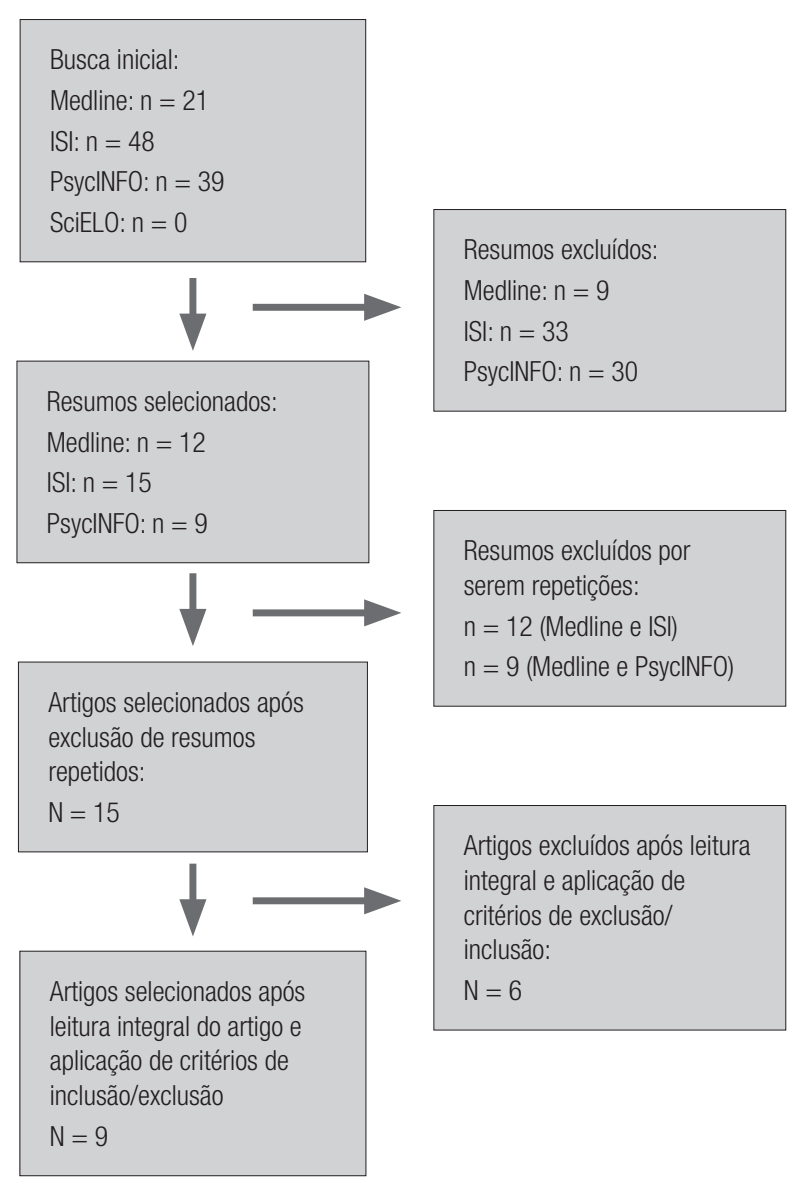

Figura 1. Fluxograma 
Tabela 2. Estudos sobre metacognição no transtorno bipolar

\begin{tabular}{|c|c|c|}
\hline Estudo & Amostra e desenho & Objetivo \\
\hline Østefjells et & 80 TB $\times 166 \mathrm{C}$ & Met: TB x C \\
\hline al., 2017 & & $\begin{array}{l}\text { Met x idade de abertura; número } \\
\text { de episódios afetivos; sintomas } \\
\text { maníacos (YMRS); sintomas } \\
\text { depressivos (IDS-C); estratégias } \\
\text { de controle de pensamentos } \\
\text { (TCQ) }\end{array}$ \\
\hline
\end{tabular}

\section{Instrumento de avaliação Resultados}

MCQ-30

; número depressivos (IDS-C); estratégias (TCQ)

\begin{tabular}{|c|c|c|}
\hline $\begin{array}{l}\text { Zhang et } \\
\text { al., } 2015\end{array}$ & $\begin{array}{l}17 \mathrm{~TB}(13 \mathrm{eu}, 4 \mathrm{dp}) \times 170 \\
\mathrm{SCH} \times 21 \mathrm{C}\end{array}$ & $\begin{array}{l}\text { Met: TB x SCH x C } \\
\text { Met x imagem por ressonância } \\
\text { magnética funcional }\end{array}$ \\
\hline $\begin{array}{l}\text { Haffner et } \\
\text { al., } 2018\end{array}$ & 34 TB eu & $\begin{array}{l}\text { TM x funcionamento psicossocial } \\
\text { (FAST); qualidade de vida } \\
\text { (WHOQOL-BREF) }\end{array}$ \\
\hline
\end{tabular}

Tarefas de autorreflexão consistindo em três condições: (autorreflexão, heterorreflexão, controle semântico)

TM

\begin{tabular}{|c|c|c|}
\hline $\begin{array}{l}\text { Tas et al., } \\
2014\end{array}$ & $30 \mathrm{~TB} \times 30 \mathrm{SCH}$ & $\begin{array}{l}\text { Met: TB x SCH } \\
\text { Met x duração da doença; idade; } \\
\text { nível educacional; funcionamento } \\
\text { executivo (WCST); memória } \\
\text { verbal }\end{array}$ \\
\hline $\begin{array}{l}\text { Lysaker et } \\
\text { al., } 2018\end{array}$ & 26 TB $\times 26$ SCH $\times 36 \mathrm{C}$ & $\begin{array}{l}\text { Met: TB x SCH x C } \\
\text { Met x sintomas positivos e } \\
\text { negativos (PANSS) }\end{array}$ \\
\hline $\begin{array}{l}\text { Batmaz et } \\
\text { al., } 2014\end{array}$ & 140 TB x 166 DM x151 C & Met: TB x DM x C \\
\hline $\begin{array}{l}\text { Sarisoy et } \\
\text { al., } 2014\end{array}$ & $45 \mathrm{~TB} d p \times 51 \mathrm{DM} d p \times 60 \mathrm{C}$ & $\begin{array}{l}\text { Met: TB x DM x C } \\
\text { Met x sintomas ansiosos (BAS); } \\
\text { sintomas depressivos (BDI); } \\
\text { autoestima (RSES) }\end{array}$ \\
\hline
\end{tabular}

MAS-A

Met (autorreflexividade): $\mathrm{TB}>\mathrm{SCH}$

$>$ Met (descentralização): > duração da doença

$>$ Met (autorreflexividade): > idade

$>$ Met (autorreflexividade): $>$ nível educacional

$>$ Met (domínio): > funcionamento executivo

$>$ Met (domínio): > memória verbal

MAS-A

Met: $\mathrm{TB}=\mathrm{C}>\mathrm{SCH}$

$<$ Met (todos exceto descentralização): > excitação

$<$ Met (total e domínio): > sintomas cognitivos

MCQ-30 Met: $\mathrm{TB}=\mathrm{DM}<\mathrm{C}$

MCQ-30 Met (controle de pensamentos e incontrolabilidade/ perigo): $\mathrm{TB}=\mathrm{DM}<\mathrm{C}$

Met (confiança cognitiva): TB $<$ C

$<$ Met (preocupação, controle de pensamentos e incontrolabilidade/perigo): > sintomas depressivos

$<$ Met (preocupação, confiança cognitiva, autoconsciência cognitiva, controle de pensamentos e incontrolabilidade/perigo): > sintomas ansiosos

$<$ Met (controle de pensamentos e incontrolabilidade/ perigo): < autoestima

\begin{tabular}{|c|c|c|c|c|}
\hline $\begin{array}{l}\text { Torres et al., } \\
2016\end{array}$ & 50 TB eu $\times 38$ C & Met: TB $\times$ C & $\begin{array}{l}\text { GR } \\
\text { PR }\end{array}$ & $\begin{array}{l}\text { Met }(G R): T B<C \\
\operatorname{Met}(P R): T B=C\end{array}$ \\
\hline $\begin{array}{l}\text { Popolo et } \\
\text { al., } 2017\end{array}$ & $23 \mathrm{~TB} \times 26 \mathrm{SCH} \times 23 \mathrm{C}$ & $\begin{array}{l}\text { Met: TB } \times \text { SCH x C } \\
\text { Met } x \text { sintomas psíquicos (BPRS) }\end{array}$ & $\begin{array}{l}\text { MAS-A } \\
\text { MCQ-30 }\end{array}$ & $\begin{array}{l}\text { Met (MAS-A total e subescalas): } \mathrm{SCH}<\mathrm{TB}<\mathrm{C} \\
\text { Met (MCQ-30): } \mathrm{SCH}=\mathrm{TB}=\mathrm{C} \\
>\text { Met (MAS-A total, autorreflexividade e domínio): }< \\
\text { retraimento/retardamento } \\
<\text { Met (MCQ-30 total e controle cognitivo): }> \\
\text { sintomas totais }\end{array}$ \\
\hline
\end{tabular}

TB: transtorno bipolar; C: controle; DM: depressão unipolar; eu: eutímico; dp: deprimidos; SCH: esquizofrenia; Met: capacidade metacognitiva; TM: treinamento metacognitivo; MCQ-30: Metacognitions Questionnaire-30 items; MAS-A: Metacognition Assessment Scale Abbreviated; GR: global metacognitive knowledge general rating; PR: global metacognitive experience postdiction rating; YMRS: Young Mania Rating Scale; IDS-C: Inventory of Depressive Symptoms - Clinician Rated; TCQ: Thought Control Questionnaire; FAST: Functional Assessment Short Test, WHOQOL-BREF: World Health Organization Quality of Life; WCST: Wisconsin Card Sorting Test, PANSS: Positive and Negative Syndrome Scale; BAS: Beck Anxiety Scale; BDI: Beck Depression Inventory; RSES: Rosenberg Self-Esteem Scale; BPRS: Brief Psychiatric Rating Scale. 


\section{Instrumentos}

A maioria dos estudos ${ }^{44-46,51-53}$ utilizou alguma escala de avaliação da metacognição.

Quatro estudos $45,51-53$ utilizaram O Metacognitions Questionnaire-30 (MCQ-30)54. O MCQ-30 é uma medida de autorrelato e contém cinco subescalas: crenças positivas sobre preocupações, crenças negativas sobre a incontrolabilidade e perigo de preocupação, confiança cognitiva, autoconsciência cognitiva e necessidade de controle.

Três estudos ${ }^{44-46}$ utilizaram o Metacognition Assessment Scale-Abbreviated (MAS-A) 55 . O MAS-A foi adaptado com base no instrumento original: a Metacognitive Assessment Scale ${ }^{56}$. O MAS-A contém quatro subescalas: autorreflexividade, que se refere à capacidade de pensar sobre si mesmo; consciência da mente do outro, que se refere à consciência dos estados mentais dos outros, tais como pensamentos e emoções; descentralização, que se refere à capacidade de ver o mundo a partir de múltiplas perspectivas; domínio, que se refere à capacidade de usar o conhecimento de si e dos outros para responder aos desafios psicológicos.

Dois estudos ${ }^{57,58}$ aplicaram tarefas para avaliação de metacognição. Zhang et al. (2015)57 utilizaram tarefas envolvendo autorreflexão, que consistiam de sentenças envolvendo o próprio paciente, tarefas de heterorreflexão, envolvendo questões sobre parentes ou amigos próximos, e tarefas de controle semântico, que envolviam questões sobre conhecimento geral. Torres et al. (2016) ${ }^{58}$ avaliaram a metacognição a partir de perguntas de autorreflexão que foram aplicadas em dois momentos: antes da realização de uma bateria de testes neuropsicológicos, que avaliou o conhecimento metacognitivo, e após a execução desses testes, avaliando a experiência metacognitiva. Um estudo ${ }^{59}$ aplicou um treinamento metacognitivo que aborda vieses cognitivos, cognição social e autoestima.

\section{Comparação entre o TB e outros transtornos mentais}

Seis estudos ${ }^{44,45,51-53,57}$ compararam bipolares com controles

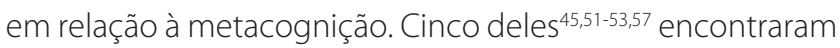
que bipolares tinham menos capacidade metacognitiva do que controles. $\mathrm{O}$ outro $^{44}$ não encontrou diferença quanto à capacidade metacognitiva entre bipolares e controles.

Um estudo ${ }^{58}$ avaliou o conhecimento metacognitivo entre bipolares e controles e encontrou que bipolares tinham menor capacidade metacognitiva do que controles. Esse mesmo estudo avaliou a experiência metacognitiva entre bipolares e controles e não encontrou diferença entre eles.

Dois estudos ${ }^{51,52}$ compararam bipolares com deprimidos unipolares em relação à metacognição e não encontraram diferença entre eles. Três estudos ${ }^{45,46,57}$ compararam bipolares com esquizofrênicos em relação à metacognição. Um deles ${ }^{57}$ não encontrou diferença entre eles. Dois outros estudos ${ }^{45,46}$ mostraram que bipolares tinham melhor capacidade metacognitiva do que esquizofrênicos.

\section{Dados sociodemográficos}

Um estudo ${ }^{46}$ encontrou que maior capacidade metacognitiva estava associada à maior idade. Esse estudo ${ }^{46}$ também relatou que maior capacidade metacognitiva estava associada a maior nível educacional.

\section{Delineamento}

\section{Dados clínicos retrospectivos}

Um estudo ${ }^{53}$ relatou que menor capacidade metacognitiva estava associada a menor idade de abertura da doença afetiva. Esse mesmo estudo ${ }^{53}$ também observou que menor capacidade metacognitiva estava associada a um maior número de episódios depressivos.

Dois estudos ${ }^{46,53}$ avaliaram a relação entre metacognição e duração da doença. Um deles ${ }^{46}$ encontrou que maior capacidade metacognitiva estava associada a maior duração da doença. O outro ${ }^{53}$ não encontrou correlação entre metacognição e duração do transtorno afetivo.

\section{Dados clínicos prospectivos}

Dois estudos ${ }^{51,53}$ encontraram que menor capacidade metacognitiva estava relacionada a maior presença de sintomas depressivos. Um estudo ${ }^{51}$ relatou que menor capacidade metacognitiva estava relacionada a maior presença de sintomas de ansiedade. E esse mesmo estudo ${ }^{51}$ encontrou que menor capacidade metacognitiva estava relacionada a menor autoestima.

A relação entre metacognição e sintomas de excitação foi avaliada por um estudo ${ }^{44}$ que encontrou que menor capacidade metacognitiva estava relacionada a maior presença de sintomas de excitação. Por sua vez, outro estudo ${ }^{53}$ relatou que menor capacidade metacognitiva estava relacionada a maior presença de sintomas maníacos.

Um estudo ${ }^{45}$ investigou a relação entre metacognição e sintomas negativos e encontrou que menor capacidade metacognitiva estava relacionada a maior presença de sintomas negativos. Outro estudo ${ }^{44}$ relatou que menor capacidade metacognitiva estava relacionada a maior presença de sintomas cognitivos, avaliados pela Positive and Negative Syndrome Scale (PANSS).

A relação entre metacognição e estratégias de uso de controle do pensamento foi avaliada por um estudo ${ }^{53}$. Østefiells et al. (2017) ${ }^{53}$ encontraram que menor capacidade metacognitiva estava relacionada a maior uso de estratégias de controle do pensamento. Outro estudo ${ }^{46}$ relatou que maior capacidade metacognitiva estava associada a melhor funcionamento executivo. Esse mesmo estudo também encontrou que maior capacidade metacognitiva estava associada a maior memória verbal. 
Apenas um estudo ${ }^{57}$ avaliou ativação cerebral versus capacidade metacognitiva no TB. Esse estudo encontrou que durante uma tarefa de heterorreflexão houve menor ativação no córtex cingulado posterior estendendo-se ao precuneus em bipolares do que em controles. E nesse estudo não houve diferenciação de ativação entre bipolares e esquizofrênicos. Esse mesmo estudo também mostrou que não houve diferenças de grupo na ativação cerebral durante a tarefa de autorreflexão.

$\mathrm{O}$ estudo ${ }^{59}$ que realizou um treinamento metacognitivo em pacientes bipolares mostrou que houve uma melhora de funcionamento psicossocial. Contudo, esse mesmo estudo não encontrou relação com uma melhora de qualidade de vida. Esse treinamento é uma intervenção psicológica para um grupo de 3 a 10 participantes. As oito sessões duram de 60 a 90 minutos. Os pacientes são treinados sobre como tomar consciência de seus padrões cognitivos, ilustrados por exemplos da vida cotidiana e de desenhos animados. Todos os tópicos são discutidos e considerados da perspectiva do afeto maníaco e depressivo. Além disso, destaca-se como as distorções cognitivas podem diferir entre os participantes. Mudanças no modo de pensar do indivíduo são destacadas como possíveis sinais de alerta para os próximos episódios. Finalmente, padrões e estratégias cognitivos mais apropriados são desenvolvidos e treinados exaustivamente usando vários exemplos diversos. No final das sessões, planilhas e exercícios de atenção são distribuídos aos participantes para levar para casa, aprimorar e consolidar seu aprendizado.

\section{DISCUSSÃO}

A presente revisão constatou que a maioria dos estudos sobre metacognição no TB - oito dos nove estudos selecionados - realizou uma comparação entre TB e outros grupos

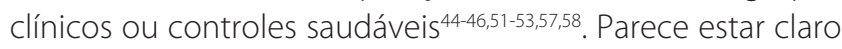
que pacientes bipolares apresentam prejuízo de metacognição quando comparados com controles saudáveis 45,51-53,57,58. A hipótese de que pacientes com TB experimentam certas formas de déficits metacognitivos, que são geralmente menos graves do que aqueles encontrados na esquizofrenia, foi corroborada com os achados dos estudos de Tas et al. $(2014)^{46}$ e Popolo et al. (2017) $)^{45}$. O estudo ${ }^{57}$ que não encontrou diferença entre esses grupos quanto a metacognição tinha uma amostra menor de bipolares, o que poderia configurar uma limitação. Apesar de poucos, os estudos parecem mostrar que não há diferença quanto ao prejuízo de capacidade metacognitiva entre depressão bipolar e unipolar ${ }^{51,52}$.

Um estudo ${ }^{46}$ mostrou que um maior nível educacional está relacionado a um maior nível de metacognição. Provavelmente os pacientes com um maior nível educacional tiveram maior acesso ao conhecimento sobre a sua doença e sobre as formas de lidar com ela. É possível também que eles possuam um maior status socioeconômico, com acesso a melhores serviços de saúde e formas de tratamento. Esse mesmo estudo também apontou que maior capacidade metacognitiva está associada à maior duração da doença, sugerindo que um maior período de convivência com a doença levaria a um melhor conhecimento sobre sua sintomatologia. Entretanto, como apenas um artigo encontrou essa relação, não é possível concluir que, de fato, o tempo de estudo ou maior duração da doença de um paciente possa ser determinante para uma melhor metacognição.

Três estudos ${ }^{44,51,53}$ apontaram que a maior presença de sintomas afetivos está associada à pior metacognição. Dois desses estudos ${ }^{51,53}$ encontraram que menor capacidade metacognitiva estava relacionada à maior presença de sintomas depressivos. Esse resultado é semelhante ao encontrado por um estudo ${ }^{60}$ conduzido com pacientes deprimidos unipolares. Por sua vez, dois estudos ${ }^{44,53}$ mostraram relação entre menor capacidade metacognitiva e maior presença de sintomas maníacos. Esses dados apontam que a presença de sintomatologia aguda pode interferir na capacidade metacognitiva do indivíduo, mas o delineamento transversal impede conclusões sobre causalidade. Além disso, nenhum estudo comparou a capacidade metacognitiva em pacientes bipolares em diversos estados afetivos.

Menor capacidade metacognitiva está relacionada a níveis mais altos de sintomas negativos em bipolares ${ }^{45}$. Esse resultado é corroborado por outros trabalhos que sugerem que os sintomas negativos podem levar a déficits nas habilidades metacognitivas ${ }^{61}$. Popolo et al. $(2017)^{45}$ sugerem que a deficiência nas habilidades de entender os estados mentais torna mais difícil para as pessoas com TB se envolverem com o mundo, o que poderia levá-los a pensar que um retraimento, um sintoma negativo, seja a opção mais viável. Contudo, por ser um estudo transversal, também não é possível estabelecer relação definitiva de causalidade.

Um estudo ${ }^{51}$ relatou que menor capacidade metacognitiva estava relacionada a maior presença de sintomas de ansiedade. Esse resultado corrobora achados de estudos sobre metacognição em transtornos ansiosos, que relataram um maior prejuízo de capacidade metacognitiva em transtorno obsessivo-compulsivo ${ }^{24,25,62}$, transtorno de pânico ${ }^{25,26}$ e transtorno de ansiedade generalizada ${ }^{26,27}$ do que em grupos de controles saudáveis. Em conjunto, esses achados sugerem que pior metacognição esteja associada a sintomas de ansiedade.

Um estudo ${ }^{53}$ apontou que pior capacidade metacognitiva está associada a maior uso de estratégias de controle do pensamento, como reavaliação, controle social e preocupação. Esse resultado corrobora os achados de pesquisa anterior sobre metacognição e maior uso de estratégias de controle do pensamento ${ }^{60}$. Apesar de o estudo de Østefjells et al. (2017) ${ }^{53}$ ser transversal, os autores sugerem que a metacognição poderia influenciar o uso de estratégias de controle do pensamento, o que poderia prejudicar a resolução de desgastes emocionais. 
Um estudo ${ }^{57}$ observou menor ativação no córtex cingulado posterior, estendendo-se ao precuneus durante heterorreflexão em pacientes com TB, em comparação com os controles saudáveis. Tanto o córtex cingulado posterior como o precuneus têm sido relatados como sendo áreas importantes subjacentes à memória autobiográfica ${ }^{63-65}$. Alguns estudos sugerem que a memória autobiográfica pode desempenhar um papel crítico no processamento autorreflexivo ${ }^{66}$ e também no processamento heterorreflexivo ${ }^{67}$. De fato, de acordo com o modelo de autorreflexão/autoavaliação ${ }^{67}$, o papel do córtex cingulado posterior e do precuneus durante a auto e a heterorreflexão está associado ao processamento da memória autobiográfica. $\mathrm{O}$ estudo de Zhang et al. $(2015)^{57}$ sugere que menos ativação no córtex cingulado posterior/precuneus reflete uma integração reduzida entre informações passadas e atuais e, mais especificamente, com relação a informações sobre outras pessoas. De acordo com essa sugestão, a literatura anterior mostrou que a memória autobiográfica é de fato prejudicada em pacientes com TB. Por exemplo, pacientes com TB fornecem menos detalhes durante a memória autobiográfica do que indivíduos saudáveis ${ }^{68,69}$.

O estudo piloto de Haffner et al. (2018)59 é o primeiro a avaliar um treinamento metacognitivo em pacientes bipolares. O estudo indicou a viabilidade desse tipo de intervenção em indivíduos com TB. Esse estudo encontrou uma melhoria do funcionamento psicossocial global. Esse efeito foi evidenciado por melhorias significativas em diferentes domínios de funcionamento, como independência e funcionamentos cognitivo, ocupacional e interpessoal. Portanto, o treinamento metacognitivo poderia ser uma boa opção de terapia adjunta a outros tratamentos. Futuramente, será importante confirmar a eficácia desse treinamento em outros estudos com amostras maiores, uma condição de controle e parâmetros de resultados de longo prazo, como tempo até recidiva e número de episódios.

O fato de apenas um pesquisador ter realizado o levantamento bibliográfico e o julgamento dos artigos a serem selecionados configura uma limitação da presente revisão sistemática. O ideal é que houvesse mais de um pesquisador nessas tarefas para minimizar possíveis vieses no processo de identificação e seleção dos documentos. A presença de poucos estudos sobre metacognição no TB permite apenas conclusões limitadas. Portanto, é um campo que ainda precisa ser bastante estudado. Nenhum estudo avaliou a influência do estado afetivo sobre a metacognição. É importante que haja mais estudos sobre intervenções metacognitivas em pacientes bipolares para avalição de viabilidade dessa prática.

\section{CONCLUSÃO}

Os estudos sobre metacognição no TB ainda são escassos. Contudo, parece estar claro que há prejuízo metacognitivo em bipolares quando comparados a controles saudáveis. Além disso, parece haver influência do estado afetivo sobre a metacognição em TB. Por sua vez, o campo sobre estudos de intervenção metacognitiva em bipolares parece ser promissor.

\section{CONTRIBUIÇÕES INDIVIDUAIS}

Rafael de Assis da Silva - Participou do desenho do estudo, da coleta, análise e seleção dos artigos e da redação do manuscrito e aprovou a versão final a ser publicada.

Marcelo Baggi Tancini - Participou do desenho do estudo, da análise dos artigos e da redação do manuscrito e aprovou a versão final a ser publicada.

Elie Cheniaux - Participou do desenho do estudo e da redação do manuscrito e aprovou a versão final a ser publicada.

Daniel C. Mograbi - Participou do desenho do estudo e da redação do manuscrito e aprovou a versão final a ser publicada.

\section{CONFLITO DE INTERESSES}

Não há conflito de interesses.

\section{AGRADECIMENTOS}

Fundação de Amparo à Pesquisa do Estado do Rio de Janeiro (Faperj).

\section{REFERÊNCIAS}

1. Mograbi DC, Brown RG, Salas CR, Morris RG. Emotional reactivity and awareness of task performance in Alzheimer's disease. Neuropsychologia. 2012;50(8):2075-84.

2. Flavell JH. Metacognition and cognitive monitoring: a new area of cognitivedevelopmental inquiry. Am Psychologist. 1979;34(10):906-11.

3. Kuhn D. Metacognitive development. Curr Dir Psychol Sci. 2000;9(5):178-81.

4. Wells A, Matthews G. Modelling cognition in emotional disorder: the S-REF model. Behav Res Ther. 1996;34(11-12):881-8.

5. Nelson TO, Stuart RB, Howard C, Crowley M. Metacognition and clinical psychology: a preliminary framework for research and practice. Clin Psychol Psychother. 1999;6:73-9.

6. Arnon-Ribenfeld N, Hasson-Ohayon I, Lavidor M, Atzil-Slonim D, Lysaker PH. The association between metacognitive abilities and outcome measures among people with schizophrenia: a meta-analysis. Eur Psychiatry. 2017;46:33-41.

7. Nabors LM, Yanos PT, Roe D, Hasson-Ohayon I, Leonhardt BL, Buck KD, et al. Stereotype endorsement, metacognitive capacity, and self-esteem as predictors of stigma resistance in persons with schizophrenia. Comp Psychiatry. 2014;55(4):792-8.

8. Kukla M, Lysaker PH, Salyers M. Do persons with schizophrenia who have better metacognitive capacity also have a stronger subjective experience of recovery? Psychiatry Res. 2013;209(3):381-5.

9. Lysaker PH, Dimaggio G, Carcione A, Procacci M, Buck KD, Davis LW, et al. Metacognition and Schizophrenia: the capacity for self- reflectivity as a predictor for prospective assessments of work performance over six months. Schizophr Res. 2010;122(1-3):124-30. 
10. Willits JA, Rubin T, Jones MH, Minor KS, Lysaker PH. Evidence of disturbances of deep levels of semantic cohesion within personal narratives in schizophrenia. Schizophr Res. 2018;197:365-9.

11. Luther $L$, Bonfils KA, Firmin RL, Buck KD, Choi J, Dimaggio G, et al. Is metacognition necessary for the emergence of motivation in schizophrenia? A necessary condition analysis. J Nerv Ment Dis. 2017;205(12):960-6.

12. Luther L, Firmin RL, Minor KS, Vohs JL, Buck B, Buck KD, et al. Metacognition deficits as a risk factor for prospective motivation deficits in schizophrenia spectrum disorders. Psychiatry Res. 2016;245:172-8.

13. Lysaker PH, Dimaggio G, Buck KD, Callaway SS, Salvatore G, Carcione A, et al. Poor insight in schizophrenia: Links between different forms of metacognition with awareness of symptoms, treatment need and consequences of illness. Compr Psychiatry. 2011;52(3):253-60.

14. Vohs JL, George S, Leonhardt BL, Lysaker PH. Impairments in insight and reflectivity in schizophrenia: Correlates, consequences and developing treatment approaches. Expert Rev Neurother. 2016;22:1-12.

15. Jansen JE, Lysaker PH, Trauelsen AM, Luther L, Haahr UH, Lyse HG, et al. Metacognitive mastery in persons with first-episode psychosis and their caregivers: Implications for timely help-seeking and caregiver experiences. Brief report. Psychiatry Res. 2017;251:54-7.

16. Lysaker PH, Vohs J, Hamm JA, Kukla M, Minor KS, de Jong S, et al. Deficits in metacognitive capacity distinguish patients with schizophrenia from those with prolonged medical adversity. J Psychiatr Res. 2014;55:126-32.

17. Vohs J, Lysaker PH, Francis M, Hamm J, Buck K, Olesek K, et al. Metacognition, social cognition, and symptoms in patients with first episode and prolonged psychoses. Schizophr Res. 2014;153(1-3):54-9.

18. Hasson-Ohayon I, Avidan M, Mashiach-Eizenberg M, Kravetz S, Rozencwaig S, Shalev $\mathrm{H}$, et al. Metacognitive and social cognition approaches to understanding the impact of schizophrenia on social quality of life. Schizophr Res. 2015;161(2-3):386-91.

19. Massé M, Lecomte T. Metacognitive profiles in individuals with a first episode of psychosis and their relation to social functioning and perceived social support. Schizophr Res. 2015;166(1-3):60-4.

20. MacBeth A, Gumley A, Schwannauer M, Carcione A, McLeod H, Dimaggio G. Metacognition in first episode psychosis: item level analysis of associations with symptoms and engagement. Clin Psychol Psychother. 2016;23(4):329-39.

21. Dimaggio G, Procacci M, Nicolò G, Popolo R, Semerari A, Carcione A, et al. Poor metacognition in narcissistic and avoidant personality disorders: four psychotherapy patients analysed using the Metacognition Assessment Scale. Clin Psychol Psychother. 2007;14(5):386-401.

22. Carcione A, Semerari A, Nicolò G, Pedone R, Popolo R, Conti L, et al. Metacognitive mastery dysfunctions in personality disorder psychotherapy. Psychiatry Res. 2011;190(1):60-71.

23. Nicolò G, Semerari A, Lysaker PH, Dimaggio G, Conti L, D’Angerio S, et al. Alexithymia in personality disorders: correlations with symptoms and interpersonal functioning. Psychiatry Res. 2011;190(1):37-42.

24. Moritz S, Peters MJ, Larøi F, Lincoln TM. Metacognitive beliefs in obsessive-compulsive patients: a comparison with healthy and schizophrenia participants. Cogn Neuropsychiatry. 2010;15(6):531-48.

25. Cucchi M, Bottelli V, Cavadini D, Ricci L, Canca V, Ronchi P, et al. An explorative study on metacognition in obsessive-compulsive disorder and panic disorder. Compr Psychiatry. 2012;53(5):546-53.

26. Morrison AP, Wells A. A comparison of metacognitions in patients with hallucinations, delusions, panic disorder, and non-patient controls. Behav Res Ther. 2003:41(2):251-6.

27. Barahmand U. Meta-cognitive profiles in anxiety disorders. Psychiatry Res. 2009;169(3):240-3.

28. PapageorgiouC,VentourasE,LykourasL,UzunogluN, ChristodoulouGN. Psychophysiological evidence for altered information processing in delusional misidentification syndromes. Prog Neuropsychopharmacol Biol Psychiatry. 2003;27(3):365-72.

29. Honkalampi K, Hintikka J, Antikainen R, Lehtonen J, Viinamäki H. Alexithymia in patients with major depressive disorder and comorbid cluster C personality disorders: a 6-month follow-up study. J Pers Disord. 2001;15(3):245-54.

30. Stefanopoulou E, Manoharan A, Landau S, Geddes JR, Goodwin G, Frangou S. Cognitive functioning in patients with affective disorders and schizophrenia: a meta-analysis. Int Rev Psychiatry. 2009;21(4):336-56
31. Yatham LN, Kauer-Sant'Anna M, Bond DJ, Lam RW, Torres I. Course and outcome after the first manic episode in patients with bipolar disorder: prospective 12-month data from the systematic treatment optimization program for early mania project. Can J Psychiatry. 2009;54(2):105-12

32. Amador XF, Flaum M, Andreasen NC, Strauss DH, Yale SA, Clark SC, et al. Awareness of illness in schizophrenia and schizoaffective and mood disorders. Arch Gen Psychiatry. 1994;51(10):826-36.

33. Silva Rde A, Mograbi DC, Bifano J, Santana CMT, Cheniaux E. Insight in bipolar mania: evaluation of its heterogeneity and correlation with clinical symptoms. J Affect Disord. 2016;199:95-8.

34. Silva RA, Mograbi DC, Landeira-Fernadez J, Cheniaux E. 0 insight no transtorno bipolar: uma revisão sistemática. J Bras Psiquiatr. 2014;63(3):242-54.

35. de Assis da Silva R, Mograbi DC, Silveira LAS, Nunes ALS, Novis FD, Landeira-Fernandez J, et al. Insight across the different mood states of bipolar disorder. Psychiatr Q. 2015;86(3):395405 .

36. Dias VV, Brissos S, Carita Al. Clinical and neurocognitive correlates of insight in patients with bipolar I disorder in remission. Acta Psychiatr Scand. 2008:117(1):28-34.

37. Varga M, Magnusson A, Flekkøy K, Rønneberg U, Opjordsmoen S. Insight, symptoms and neurocognition in bipolar I patients. J Affect Disord. 2006;91(1):1-9.

38. Wolf F, Brüne M, Assion HJ. Theory of mind and neurocognitive functioning in patients with bipolar disorder. Bipolar Disord. 2010;12(6):657-66.

39. Martino DJ, Strejilevich SA, Fassi G, Marengo E, Igoa A. Theory of mind and facial emotion recognition in euthymic bipolar I and bipolar II disorders. Psychiatry Res. 2011;189(3):379-84.

40. Benito A, Lahera G, Herrera S, Muncharaz R, Benito G, Fernández-Liria A, et al. Deficits in recognition, identification, and discrimination of facial emotions in patients with bipolar disorder. Rev Bras Psiquiatr. 2013;35(4):435-8.

41. Temmerman A, Sabbe B, Morrens M. [Social cognition in bipolar disorder]. Tijdschr Psychiatr. 2015;57(6):405-14

42. Santos JM, Pousa E, Soto E, Comes A, Roura P, Arrufat FX, et al. Theory of mind in euthymic bipolar patients and first-degree relatives. J Nerv Ment Dis. 2017;205(3):207-12.

43. Ioannidi N, Konstantakopolous G, Sakkas D, Oulis P. The relationship of Theory of Mind with symptoms and cognitive impairment in bipolar disorder: a prospective study. Psychiatriki. 2015;26(1):17-27.

44. Lysaker PH, Irarrázaval L, Gagen EC, Armijo I, Ballerini M, Mancini M, et al. Metacognition in schizophrenia disorders: Comparisons with community controls and bipolar disorder: Replication with a Spanish language Chilean sample. Psychiatry Res. 2018;267:528-34.

45. Popolo R, Smith E, Lysaker PH, Lesting K, Cavallo F, Melchiorre L, et al. Metacognitive profiles in schizophrenia and bipolar disorder: comparisons with healthy controls and correlations with negative symptoms. Psychiatry Res. 2017;257:45-50.

46. Tas C, Brown EC, Aydemir 0, Brüne M, Lysaker PH. Metacognition in psychosis: comparison of schizophrenia with bipolar disorder. Psychiatry Res. 2014;219(3):464-9.

47. Lysaker PH, Kukla M, Belanger E, White DA, Buck KD, Luther L, et al. Individual psychotherapy and changes in self-experience in schizophrenia: a qualitative comparison of patients in metacognitively focused and supportive psychotherapy. Psychiatry. 2015;78(4):305-16.

48 Dimaggio G, Montano A, Popolo R, Salvatore G. Metacognitive interpersonal therapy for personality disorders: a treatment manual. Routledge: New York; 2015.

49. Moher D, Liberati A, Tetzlaff J, Altman DG; PRISMA Group. Preferred reporting items for systematic reviews and meta-analyses: the PRISMA statement. PLoS Med. 2009;6(7):e1000097.

50. Higgins JPT, Thomas J, Chandler J, Cumpston M, Li T, Page MJ, et al. eds. Cochrane Handbook for Systematic Reviews of Interventions version 6.0 (updated July 2019). Cochrane. 2019. Disponível em: www.training.cochrane.org/handbook.

51. Sarisoy G, Pazvantoğlu 0, Ozturan DD, Ay ND, Yilman T, Mor S, et al. Metacognitive beliefs in unipolar and bipolar depression: a comparative study. Nord J Psychiatry. 2014;68(4):275-81

52. Batmaz S, Ulusoy Kaymak S, Kocbiyik S, Turkcapar MH. Metacognitions and emotional schemas: a new cognitive perspective for the distinction between unipolar and bipolar depression. Compr Psychiatry. 2014;55(7):1546-55.

53. Østefells T, Melle I, Aminoff SR, Hellvin T, Hagen R, Lagerberg TV, et al. An exploration of metacognitive beliefs and thought control strategies in bipolar disorder. Compr Psychiatry. 2017:73:84-92. 
54. Wells A, Cartwright-Hatton S. A short form of the metacognitions questionnaire: properties of the MCQ-30. Behav Res Ther. 2004;42(4):385-96.

55. Lysaker PH, Carcione A, DiMaggio G, Johannesen JK, Nicolò G, Procacci, et al. Metacognition amidst narratives of self and illness in schizophrenia: associations with neurocognition, symptoms, insight and quality of life. Acta Psychiatr Scand. 2005;112(1):64-71.

56. Semerari A, Carcione A, Dimaggio G, Falcone M, Nicolo G, Procaci M, et al. How to evaluate metacognitive function in psychotherapy? The metacognition assessment scale and its applications. Clin Psychol Psychother. 2003;10(4):238-61.

57. Zhang L, Opmeer EM, Ruhé HG, Aleman A, van der Meer L. Brain activation during self- and other-reflection in bipolar disorder with a history of psychosis: comparison to schizophrenia. Neuroimage Clin. 2015;8:202-9.

58. Torres IJ, Mackala SA, Kozicky JM, Yatham LN. Metacognitive knowledge and experience in recently diagnosed patients with bipolar disorder. J Clin Exp Neuropsychol. 2016;38(7):730-44.

59. Haffner P, Quinlivan E, Fiebig J, Sondergeld LM, Strasser ES, Adli M, et al. Improving functional outcome in bipolar disorder: A pilot study on metacognitive training. Clin Psychol Psychother. 2018;25(1):50-8.

60. Halvorsen M, Hagen R, Hjemdal 0, Eriksen MS, Sørli ÅJ, Waterloo K, et al. Metacognitions and thought control strategies in unipolar major depression: a comparison of currently depressed, previously depressed, and never-depressed individuals. Cogn Ther Res. 2014:39:31-40.

61. Buck KD, McLeod HJ, Gumley A, Dimaggio G, Buck BE, Minor KS, et al. Anhedonia in prolonged schizophrenia spectrum patients with relatively lower vs. higher levels of depression disorders: associations with deficits in social cognition and metacognition. Conscious Cogn. 2014;29:68-75.
62. Hermans D, Engelen U, Grouwels L, Joos E, Lemmens J, Pieters G. Cognitive confidence in obsessive-compulsive disorder: Distrusting perception, attention and memory. Behav Res Ther. 2008:46(1):98-113.

63. Fink GR, Markowitsch HJ, Reinkemeier M, Bruckbauer T, Kessler J, Heiss WD. Cerebral representation of one's own past: neural networks involved in autobiographical memory. J. Neurosci. 1996;16(13):4275-82.

64. Maddock RJ, Garrett AS, Buonocore MH. Remembering familiar people: the posterior cingulate cortex and autobiographical memory retrieval. Neuroscience. 2001;104(3): $667-76$.

65. Maguire EA., Mummery (J. Differential modulation of a common memory retrieval network revealed by positron emission tomography. Hippocampus. 1999;9(1):54-61.

66. Dimaggio G, Vanheule S, Lysaker PH, Carcione A, Nicolò G. Impaired self-reflection in psychiatric disorders among adults: a proposal for the existence of a network of semi independent functions. Conscious Cogn. 2009;18(3):653-64.

67. Van der Meer L, Costafreda S, Aleman A, David AS. Self-reflection and the brain: a theoretical review and meta-analysis of neuroimaging studies with implications for schizophrenia. Neurosci Biobehav Rev. 2010;34(6):935-46.

68. Scott J, Stanton B, Garland A, Ferrier IN. Cognitive vulnerability in patients with bipolar disorder. Psychol Med. 2000;30(2):467-72.

69. Shimizu M, Kubota Y, Mason R, Baba H, Calabrese JR, Toichi M. Selective deficit of autobiographical incident memory in subjects with bipolar disorder. Psychopathology. 2009:42(5):318-24. 